\title{
SÍFILIS CONGÊNITA: UM INDICADOR DA QUALIDADE DA ATENÇÃO PRIMÁRIA À SAÚDE NO ESTADO DO ACRE EM 2017
}

Rafael Tavares Lima Izel ${ }^{1}$, Jennifer Souza Maia ${ }^{1}$, Suena Ricardo de Oliveira ${ }^{1}$, Eder Ferreira de Arruda ${ }^{2}$

${ }^{1}$ Bacharel em Enfermagem pelo Centro Universitário UNINORTE, Rio Branco-AC, Brasil. E-mail: fael160@hotmail.com

${ }^{2}$ Docente do Centro Universitário UNINORTE, Rio Branco-AC, Brasil.

Recebido em: 06/04/2019 - Aprovado em: 10/06/2019 - Publicado em: 30/06/2019 DOI: 10.18677/EnciBio_2019A194

\begin{abstract}
A sífilis é uma doença sistêmica que por meio da transmissão vertical pode atingir o feto causando a sífilis congênita (SC) que se configura como um relevante problema de saúde pública, por isso o objetivo deste estudo foi descrever o perfil epidemiológico da SC no estado do Acre no ano de 2017 relacionando-o como indicador de assistência da atenção primária à saúde. Realizou-se em um estudo descritivo do tipo transversal no qual foi utilizada para a coleta de dados a base de dados do SINAN através do DATASUS. Observou-se que no estado do Acre ocorreram 81 casos de SC, com incidência geral de 4,84/1.000 nascidos vivos. Na maior parte dos casos de SC, as gestantes tinham baixa escolaridade $(48,15 \%)$, residiam na zona urbana $(67,9 \%)$, realizaram o pré-natal $(82,72 \%)$, tiveram 0 diagnóstico no pré-natal $(48,15 \%)$ e não houve tratamento dos parceiros $(65,43 \%)$. A maioria dos recém-nascidos foi diagnosticada com SC recente $(80,25 \%)$, antes dos 6 dias de vida $(96,30 \%)$, com predomínio do sexo feminino $(62,96 \%)$ e da cor parda (93,3\%). Desse modo, a SC configurou-se como um relevante problema de saúde no estado do Acre, indicando que a atenção primária não está sendo resolutiva e eficiente no controle da doença e na assistência à gestante, principalmente no interior do estado.
\end{abstract}

RESUMO

PALAVRAS-CHAVE: Atenção primária; Gestante; Sífilis congênita.

\section{CONGENITAL SYPHILIS: A QUALITY INDICATOR OF PRIMARY HEALTH CARE IN THE STATE OF ACRE IN 2017}

\begin{abstract}
Syphilis is a systemic disease that, through vertical transmission, can reach the fetus causing congenital syphilis (SC), which is a relevant public health problem, so the purpose of this study was to describe the epidemiological profile of SC in the state of Acre in the year 2017 relating it as an indicator of primary health care assistance. A descriptive cross-sectional study was carried out in which the SINAN database was used to collect data through DATASUS. It was observed that in the state of Acre there were 81 cases of SC, with a general incidence of 4.84 / 1,000 live births. In the majority of SC cases, pregnant women had low schooling $(48.15 \%)$, resided in the urban area $(67.9 \%)$, had prenatal care $(82.72 \%)$, had a prenatal diagnosis $(48.15 \%)$
\end{abstract}


and there was no treatment of the partners (65.43\%). The majority of newborns were diagnosed with a recent SC $(80.25 \%)$, before 6 days of age $(96.30 \%)$, in which the predominance was female (62.96\%) and brown (93.3\%). Thus, SC has emerged as a relevant health problem in the state of Acre, indicating that primary care is not being resolutive and efficient in controlling the disease and assisting pregnant women, especially in the state.

KEYWORDS: Primary care; Pregnant; Congenital syphilis.

\section{INTRODUÇÃO}

A sífilis é uma doença sistêmica, de evolução crônica, causado pelo Treponema pallidum, com transmissão sexual ou vertical, podendo ter períodos agudos e latentes. É classificada em sífilis adquirida recente: primária, secundária e latente recente, quando se tem menos de um ano de evolução; e sífilis adquirida tardia: latente tardia e terciária, quando se tem mais de um ano de evolução de modo que o indivíduo pode se infectar novamente toda vez que se expor ao agente (BRASIL, 2014).

A sífilis congênita (SC) ocorre devido à presença do T. pallidum na corrente sanguínea da gestante, que atravessa a placenta e atinge o feto. Embora menos frequente, o contato do recém-nascido $(R N)$ com lesões genitais também pode transmitir a sífilis. Essa infecção vertical pode ocorrer em qualquer fase da gestação, tendo o risco elevado em gestante com sífilis primária ou secundária não tratada ou tratada de maneira inadequada. Apresenta-se em dois estágios: precoce, quando diagnosticado até dois anos de vida e tardio quando diagnosticado após dois anos de idade (BRASIL, 2016).

A SC é uma doença de notificação compulsório sendo um importante indicador de assistência eficiente à gestante, sua ocorrência evidencia falhas graves no prénatal. Mesmo sendo fácil de diagnosticar e controlar, com medidas simples e de baixo custo, continua sendo um grande problema de saúde publica (BRASIL, 2010).

Essa infecção na gestação pode trazer algumas consequências para o feto, tais como: prematuridade, aborto espontâneo e óbito fetal. Pode também nascer sintomáticos ou assintomáticos, sendo o último mais frequente. Crianças assintomáticas não tratadas podem ter manifestações tardias, podendo ser irreversíveis. Pode provocar alterações ósseas e articulares, surdez, alterações dentárias, lesões oculares, nariz em cela, perfuração do palato duro, entre outras (BRASIL, 2015).

De acordo com a Portaria no 221 de 17 de abril 2008, do Ministério da Saúde (MS), a SC é um importante agravo, sendo considerada uma condição de saúde sensível à atenção primária. Neste sentido, os casos de SC podem ser considerados como um indicador da resolutividade, qualidade e acessibilidade aos serviços e ações de saúde da atenção primária (BRASIL, 2008).

Entre 2010 e 2016 as notificações de casos no Brasil passaram de 6.946 $(2,4 \%)$ para $20.474(6,8 \%)$. Na região norte no mesmo período as notificações passaram de $638(2,1 \%)$ casos para $1.724(5,4)$. No estado do acre nesse período passou de $18(1,1 \%)$ para $66(3,9 \%)$ de casos de SC. Em 2014 foram notificados 90 $(5,3 \%)$ casos, sendo o ano de maior índice de SC no Acre. Na capital do Acre, na cidade de Rio Branco, nesse mesmo período passaram de $9(1,4 \%)$ casos para 20 $(2,9 \%)$ casos. Em 2014 foram notificados 49 (7,0\%) casos, sendo o ano que mais teve casos em Rio Branco (BRASIL, 2017).

Devido a considerável importância epidemiológica da SC, seus fatores associados, possíveis desfechos e os poucos estudos sobre casos confirmados de 
SC no estado do Acre, torna-se necessário o adequado conhecimento sobre a morbidade hospitalar de crianças por esta condição para se estabelecer um melhor planejamento das ações e serviços de atenção primária e hospitalar à saúde visando à promoção, prevenção e controle deste agravo na população.

Dado o exposto, a presente pesquisa teve como objetivo descrever o perfil epidemiológico da SC no estado do Acre no ano de 2017 relacionando-o como indicador de assistência da atenção primária.

\section{MATERIAL E MÉTODOS}

O presente estudo se caracteriza como um estudo descritivo do tipo transversal. A população de estudo foram todos os 81 recém-nascidos confirmados com SC no estado do Acre no ano de 2017. Os dados referentes aos casos de SC foram coletados no mês de julho de 2018 através dos dados do Sistema de Informação de Agravos e Notificação (SINAN) disponível no sítio eletrônico do DATASUS.

Foram incluídos todos os casos confirmados no referido período, incluindo as características do recém-nascido: sexo, faixa etária, raça e classificação final da SC e as características maternas: escolaridade, zona de moradia, realização do prénatal, diagnóstico de sífilis materna e tratamento do parceiro.

A estimativa de nascidos vivos do ano de 2017 foi calculada através da média aritmética de nascidos vivos entre os anos de 2010 a 2016, usando dados do Sistema de Informações sobre Nascidos Vivos (SINASC) disponíveis no sítio eletrônico do DATASUS.

Foram excluídos da pesquisa os casos ignorados pelo sistema e casos notificados que não pertencem a este período de tempo. Também foi excluída da pesquisa a variável de faixa etária da mãe, tendo em vista que os dados disponíveis pelo sistema estavam incompletos. Para análise e tratamento dos dados, utilizou-se o programa Microsoft $\AA$ Office Excel 2010, no qual foi calculada a frequência relativa (\%) das variáveis de interesse e a incidência por 1.000 nascidos vivos.

\section{RESULTADOS E DISCUSSÃO}

No Acre foram registrados 81 casos de SC em 2017, resultado superior ao encontrado no estudo de Rosas e Nogueira (2017) que verificaram a ocorrência de 49 casos de SC em 2009 e 35 casos de SC em 2013 no estado do Acre (Tabela 1). Em relação ao município de residência, Rio Branco, a capital do Acre, apresentou a maior frequência de SC (19,75\%), em segundo lugar, Tarauacá (12,35\%), seguidos de Porto Acre, Sena Madureira e Senador Guiomard, todos com 9,88\% (Tabela 1).

De forma semelhante, na pesquisa de Guimarães et al. (2018) realizada no estado do Maranhão, identificou que em relação ao município de residência no período de 2009 a 2013 houve maior número de casos de SC na capital, São Luís $(23,0 \%)$, seguido por Imperatriz $(19,3 \%)$, segunda cidade mais populosa do Maranhão.

A incidência geral de SC no estado do Acre em 2017 foi 4,84 casos por 1.000 nascidos vivos (tabela 1). De forma diferente, no estudo de Rosas e Nogueira (2017) as incidências gerais de SC encontradas no estado do Acre em anos anteriores foram menores que a registrada neste estudo, sendo de 4,1/1.000 nascidos vivos em 2009 e de 3,6/1.000 nascidos vivos em 2013, dessa forma é possível perceber que houve um aumento da incidência de SC no estado do Acre entre os anos de 2013 e 2017. Semelhantemente, Costa et al. (2013) em pesquisa realizada no 
estado do Ceará, também identificaram um aumento na incidência de SC entre os anos de 2000 a 2009, a qual passou de 0,56 para 4,93 por 1.000 nascidos vivos.

Contudo, outros estudos identificaram uma tendência de redução na notificação de SC. Carvalho e Brito (2014) ao realizarem um estudo no estado do Rio Grande do Norte verificaram uma diminuição da incidência de SC que passou de 4,3/1.000 nascidos vivos em 2008 para 0,9/1.000 nascidos vivos em 2010. Pode-se observar também uma redução acentuada no estudo de Figueiró-Filho et al. (2012) no qual comprovaram que em Campo Grande - MS a incidência de SC passou de 23,4/1.000 nascidos vivos em 2006 para 5,85/1.000 em 2011.

No que se refere aos municípios, Porto Acre apresentou a maior incidência de SC (22,35/1.000 nascidos vivos) e Rio Branco teve a menor incidência do estado (2,34/1.000 nascidos vivos), conforme tabela 1. Diferentemente, Moreira et al. (2017) verificaram em Porto Velho - RO no ano de 2014 a incidência de SC de 8,65/1.000 nascidos vivos, número maior do que encontrado na capital do Acre.

TABELA 1 - Incidência de SC por município de residência no estado do Acre, Brasil, 2017.

\begin{tabular}{ccccc}
\hline $\begin{array}{c}\text { Município de } \\
\text { residência }\end{array}$ & $\mathbf{N}$ & $\%$ & $\begin{array}{c}\text { No estimado } \\
\text { de nascidos } \\
\text { vivos }\end{array}$ & $\begin{array}{c}\text { Incidência de SC } \\
\text { Por 1.000 } \\
\text { Nascidos Vivos }\end{array}$ \\
\hline Acrelândia & 2 & 2,47 & 244 & 8,20 \\
Assis Brasil & 1 & 1,23 & 211 & 4,74 \\
Brasiléia & 3 & 3,70 & 521 & 5,76 \\
Bujari & 3 & 3,70 & 228 & 13,16 \\
Capixaba & 3 & 3,70 & 208 & 14,42 \\
Cruzeiro do Sul & 6 & 7,41 & 1763 & 3,40 \\
Epitaciolândia & 3 & 3,70 & 274 & 10,95 \\
Feijó & 2 & 2,47 & 826 & 2,42 \\
Jordão & 2 & 2,47 & 272 & 7,35 \\
Mâncio Lima & 2 & 2,47 & 384 & 5,21 \\
Manoel Urbano & 1 & 1,23 & 246 & 4,07 \\
Porto Acre & 8 & 9,88 & 358 & 22,35 \\
Rio Branco & 16 & 19,75 & 6847 & 2,34 \\
Sena Madureira & 8 & 9,88 & 854 & 9,37 \\
Senador Guiomard & 8 & 9,88 & 425 & 18,82 \\
Tarauacá & 10 & 12,35 & 1154 & 8,67 \\
Xapuri & 3 & 3,70 & 312 & 9,62 \\
\hline Total Geral (Acre) & $\mathbf{8 1}$ & $\mathbf{1 0 0}$ & $\mathbf{1 6 7 3 3}$ & $\mathbf{4 , 8 4}$ \\
\hline
\end{tabular}

Fonte: Sistema de Informação de Agravos de Notificação, 2018.

Segundo boletim epidemiológico de 2017, a taxa nacional de SC do ano de 2016 foi de 6,8/1.000 nascidos vivos (BRASIL, 2017), desta forma o Acre encontrase abaixo da taxa nacional da doença. Porém, nem o Brasil e tampouco o Acre atingiram os objetivos do milênio estipulado pelo MS que tinham como meta eliminar a SC até 2015 (0,5/1.000 nascidos vivos). Apesar da SC no estado do Acre encontrar-se abaixo da taxa nacional, ainda está acima da meta estipulada (BRASIL, 2011).

No que se refere à escolaridade materna, a maioria das mães que tiveram filhos diagnosticados com SC tinham estudado apenas o ensino fundamental $(48,15 \%)$, conforme tabela 2 . Resultado semelhante foi encontrado no estudo 
realizado por Alves et al. (2016) em Alagoas no período de 2007 a 2011 onde 67,4\% dos casos de SC, as mães também tinham somente o ensino fundamental, assim como no estudo de Carvalho e Brito (2014) que detectaram 65\% das mulheres que tiveram filhos com SC estudaram até o ensino fundamental. Já Lafetá et al. (2016) em pesquisa realizada em Minas Gerais entre 2007 e 2013, encontraram o oposto, quando a maior parte dos casos de SC, as gestantes tinham ensino médio ou superior $(44,1 \%)$.

Esse alto número associado à baixa escolaridade encontrado na maioria dos estudos, pode estar associado com as desigualdades nas condições de saúde e acesso aos cuidados. Consequentemente a gestante tem pouco acesso às informações necessárias para evitar a infecção e a transmissão vertical, como também desconhecem a importância do tratamento completo. Em contrapartida, alguns estudos em que há predomínio de mães com nível de escolaridade elevado, reafirmam que a sífilis não afeta somente um grupo de risco, logo a prevenção deve ser para a população em geral (CARVALHO; BRITO, 2014; CUNHA, 2015).

Em relação à zona de moradia foi verificado em 2017, segundo a tabela 2 , que a maior parte das mães residia na zona urbana (67,9\%). Este resultado reforça o perfil da SC no Brasil, no qual $87 \%$ a $90 \%$ dos casos de SC as mães residem na zona urbana (ARAÚJO et al., 2011). O estudo de Figueiró-Filho et al. (2012) corrobora com esses achados, no qual detectaram que $91,32 \%$ das ocorrências de SC, as mães residiam na zona urbana e em 2011 esse número se elevou para $95,3 \%$.

Observou-se que $82,72 \%$ dos casos de SC, as mães realizaram o acompanhamento do pré-natal (Tabela 2). Característica semelhante foi verificada na pesquisa de Pereira et al. (2015) realizada na cidade de Belém, no período entre 2008 e 2013, em que 66,6\% das gestantes que tiveram filhos diagnosticados com $\mathrm{SC}$ realizaram acompanhamento pré-natal. Fato esse também encontrado no estudo de Signor et al. (2018) realizado entre 2007 e 2013 no Paraná, que identificaram que $86 \%$ dos casos de SC, as gestantes realizaram pré-natal. Semelhantemente, Alves et. al. (2016) detectaram em estudo o predomínio de $68,4 \%$ de casos de SC em que as gestantes realizaram o referido acompanhamento.

A assistência do pré-natal é de suma importância, pois tem o objetivo de identificar precocemente possíveis intercorrências danosas durante a gestação, incluindo a sífilis gestacional que pode evoluir para SC, através de exames de rotinas e testes rápidos, favorecendo a redução de riscos à saúde da gestante e do feto. Tem-se como indicador de qualidade a adesão da gestante ao pré-natal no $1^{\circ}$ 은 trimestre de gestação, na qual é o momento mais importante, pois se podem detectar tais intercorrências de forma precoce, fazer o tratamento em tempo oportuno e assim evitar a transmissão vertical para o feto (SILVA et al., 2017).

No que tange ao diagnóstico materno, a maioria dos casos da doença foi detectado durante o pré-natal $(48,15 \%)$, conforme tabela 2 . De maneira parecida, o estudo de Magalhães et al. (2013) realizado em Brasília entre os anos de 2009 a 2010, também verificou que a maioria das ocorrências de SC $(77,6 \%)$ teve o diagnóstico da doença materna durante o pré-natal. Assim como Moreira et al. (2017) verificaram que a maior parte dos casos de SC também teve o diagnóstico da doença na mãe durante o pré-natal $(60,1 \%)$.

Todavia, Lafetá et al. (2016) em pesquisa evidenciaram que $62,4 \%$ dos casos de SC, às gestantes foram diagnosticados com a doença no momento do parto ou da curetagem. Semelhantemente Guimarães et al. (2018) encontraram 52,6\% dos acontecimentos de SC em mães que também foram diagnosticadas nesse momento. 
O diagnóstico tardio da sífilis na gestação ocorre devido a várias barreiras durante o pré-natal, como a falta de acolhimento e de agilidade nos serviços, fazendo com que as gestantes demorem a aderir ao pré-natal e também a realizar os testes de rotina para diagnósticos. Situações como testes rápidos indisponíveis nas unidades, exames de rotinas com limitações mensais e o pouco contato que o profissional tem com os protocolos assistenciais, dificultando assim o manejo clínico da sífilis, também são barreiras que dificultam o diagnóstico precoce da doença (SUTO et al., 2016; GUANABARA et al., 2017).

Com relação ao parceiro, foi observado que a maior parte não realizou o tratamento para a doença $(65,43 \%)$, conforme tabela 2 . Na pesquisa de Alves et al. (2016) identificaram que $65,8 \%$ dos casos notificados de SC, os parceiros das gestantes também não realizaram o tratamento para sífilis. De forma semelhante, Silva et al. (2017) observaram em estudo que $56,86 \%$ dos eventos de SC, os parceiros da mesma forma não realizaram o tratamento para sífilis.

TABELA 2 - Características maternas dos recém-nascidos confirmados com SC no estado do Acre, Brasil, 2017.

\begin{tabular}{lcc}
\hline Variáveis & $\mathbf{N}$ & $\%$ \\
\hline Escolaridade & 2 & 2,47 \\
Analfabeto & 39 & 48,15 \\
Ensino Fundamental & 21 & 25,93 \\
Ensino Médio & 4 & 4,93 \\
Ensino Superior & 15 & 18,52 \\
Não informado & & \\
Zona de moradia & 55 & 67,9 \\
Urbana & 23 & 28,4 \\
Rural & 3 & 3,70 \\
Não informado & & \\
Realização do Pré-natal & 67 & 82,72 \\
Sim & 12 & 14,81 \\
Não & 2 & 2,47 \\
Não informado & & 48,15 \\
Diagnóstico da Sífilis & 39 & 30,86 \\
Pré-natal & 25 & 16,05 \\
Curetagem/ Parto & 13 & 1,23 \\
Após o parto & 1 & 3,70 \\
Não realizado & 3 & 23,46 \\
Não informado & & 65,43 \\
Tratamento do parceiro & 19 & 11,11 \\
Sim & 53 & \\
Não & 9 & \\
Não informado & & \\
\hline Fonte: Sistema de & & \\
\hline
\end{tabular}

Fonte: Sistema de Informação de Agravos de Notificação, (2018).

A dificuldade do tratamento do parceiro sexual da gestante diagnosticada com sífilis pode estar relacionada a não propagação de informações das mesmas aos parceiros sobre o diagnóstico positivo, devido elas não saberem da importância do tratamento do parceiro. Quando as gestantes informam a eles, há ainda uma visão ultrapassada dos homens em relação à saúde, na qual atribui a responsabilidade do 
cuidado à mulher, dificultando assim, a adesão dos parceiros aos tratamentos de saúde, incluindo o tratamento de sífilis (CAMPOS et al., 2012).

Para o MS os parceiros devem realizar o pré-natal junto com a gestante, quando não possível, deve ser chamado para a realização do tratamento, e mesmo que este não compareça, deve-se fazer a busca ativa na área de abrangência de sua moradia através da equipe saúde da família, considerando-o portador da mesma doença, devendo então receber o tratamento (BRASIL, 2015).

A elevada incidência de SC no Acre no ano de 2017 indica que existem falhas na rede de atenção primária, havendo necessidade de ações de promoção e educação em saúde à população voltada para o seu controle, tendo como meta tratar uma doença que pode ser evitada, desde que seja feito o diagnóstico de forma precoce e oportuna, mas também a conscientização da gestante infectada e seu parceiro para a importância da execução do tratamento de forma adequado. Destacando-se assim a responsabilidade elevada da atenção primária para o controle da doença (SONDA et al., 2013).

A maior parte dos RNs diagnosticados com SC era do sexo feminino, representando 62,96\% dos casos (Tabela 3). No estudo de Silva et al. (2017), semelhantemente, constataram que $64 \%$ dos casos de SC também eram do sexo feminino. O oposto foi encontrado no estudo de Silva et al. (2019) realizado em Ipojuca-PE entre os anos de 2011 a 2015, no qual foi constatado que $52,08 \%$ dos casos de SC eram do sexo masculino.

O sexo do RN não tem correlação com a infecção de SC, pois qualquer sexo esta suscetível. Logo a identificação precoce através do pré-natal de qualidade, com adesão das gestantes ao tratamento adequado é o que determina os riscos aos quais o feto estará exposto (SILVA et al., 2017).

Nesse estudo foi constatado que a maioria dos RNs foram diagnosticados com SC até o 6ํㅓㅁ dia de vida (96,30\%), conforme tabela 3. De forma igual Guimarães et al. (2018) em observaram que $94,1 \%$ dos casos de SC também foram diagnosticados até os seis dias de vida. Esse diagnóstico precoce é de suma importância, pois com o tratamento realizado o mais rápido possível, evita-se que o $\mathrm{RN}$ tenha alterações ósseas e articulares, surdez, alterações dentárias, lesões oculares, nariz em cela, perfuração do palato duro, entre outras alterações. Deve-se fazer um exame detalhado, pois é mais frequente o RN ser assintomático (SÃO PAULO, 2016).

A maioria dos casos de SC foi percebida em 93,3\% dos RNs e eram da cor parda (Tabela 3). De forma semelhante Holanda et al. (2011) observaram também que a maioria dos casos de SC eram pardos (78,1\%). Esses achados corroboram com um estudo nacional que, semelhantemente, foi identificado um predomínio da cor parda (CUNHA; HAMANN, 2015).

Já em relação à classificação da doença, $80,25 \%$ dos $\mathrm{RNs}$ tiveram SC recente e em 17,28\% dos casos este dado não foi informado (Tabela 3). Semelhantemente em seu estudo, Rosas e Nogueira (2017) observaram que em 2009, 68,6\% dos casos de SC foram classificados como SC recente e em 2013 esse número se elevou para $75 \%$. Com relação ao elevado número de dados não informados sobre a classificação da doença, essa situação pode estar relacionada à falta de conhecimento dos profissionais sobre a classificação da SC, que reflete no preenchimento das notificações, como também equívocos na hora do preenchimento das fichas de notificação e/ou na digitação dos dados (DOMINGUES et al., 2013). 
TABELA 3 - Características dos recém-nascidos com sífilis congênita no estado do Acre, Brasil, 2017.

\begin{tabular}{lcc}
\hline Variáveis & N & $\%$ \\
\hline Sexo & 30 & \\
Masculino & 51 & 37,04 \\
Feminino & & 62,96 \\
Faixa etária & 78 & 96,30 \\
Até 6 dias & 3 & 3,70 \\
27 dias ou mais & & \\
Raça & 4 & 4,94 \\
Branca & 76 & 93,83 \\
Parda & 1 & 1,23 \\
Indígena & & \\
Classificação final & 65 & 80,25 \\
Sífilis congênita recente & 2 & 2,47 \\
Descartado & 14 & 17,28 \\
Não informado &
\end{tabular}

Fonte: Sistema de Informação de Agravos de Notificação, 2018.

\section{CONCLUSÕES}

Os achados evidenciam que há grandes falhas na atenção primária, principalmente no pré-natal mesmo sendo uma doença de fácil diagnóstico, controle e com baixo custo, na qual as maiorias das gestantes realizam o pré-natal, continua ainda, sendo um relevante problema de saúde no estado do Acre.

Portanto, a atenção primária no estado do Acre não está sendo resolutiva e eficiente no controle da doença e na assistência as gestantes, dessa forma necessita-se de ações e serviços de prevenção e controle da doença.

Deve-se aproximar os profissionais de saúde com os protocolos do Ministério da Saúde, através de educação continuada e capacitação dos mesmos. Como também a gestão estadual juntamente com a gestão municipal deve proporcionar um cenário favorável ao diagnóstico precoce e ao tratamento, principalmente no interior do estado, disponibilizando os testes rápidos e, quando necessário, os exames de VDRL, como também o tratamento acessível à população.

A aproximação da comunidade com a atenção primária deve ser estreitada, favorecendo a captação precoce das gestantes e a incorporação do parceiro ao prénatal, realizando, no mínimo, seis consultas, com realização de VDRL no primeiro e no último trimestre da gestação. Como também um acolhimento de qualidade com escuta qualificada, informando, educando e esclarecendo todas as dúvidas que surgirem na gestação, melhorando assim, a adesão da gestante e parceiro ao prénatal, concomitantemente aos tratamentos que forem necessários.

\section{REFERÊNCIAS}

ALVES, W. A.; CAVALCANTI, G.R.; NUNES, F.A.; TEODORO W.R.; CARVALHO, L.M. et al. Sífilis Congênita: Epidemiologia dos Casos Notificados em Alagoas, Brasil, 2007 a 2011. Revista Portal: Saúde e Sociedade, Alagoas, v. 1, n. 1, p. 2741 , 2016.

Disponível em: http://www.seer.ufal.br/index.php/nuspfamed/article/download/2375/2192.>

ARAÚJO, C.L.; SHIMIZU, H.E; SOUSA, A. I. A; HAMANN, E.M. Incidência da sífilis congênita no Brasil e sua relação com a Estratégia Saúde da Família. Revista 
Saúde Pública, Brasília, v. 46, n. 3, p. 479-486, 2011. Disponível em: < http://www.scielo.br/pdf/rsp/v46n3/3477.pdf>.

BRASIL. Boletim epidemiológico: Sífilis. Brasília: Ministério da Saúde, 2017. 44p.

Gestação de alto risco: manual técnico. $5^{\text {a }}$ ed. Brasília: Ministério da Saúde, 2010. 302p.

.Guia de vigilância em saúde. Brasília: Ministério da Saúde, 2014. 812p.

Saúde, 2016. 54p.

Manual técnico para diagnóstico da Sífilis. Brasília: Ministério da

Ministério da Saúde. Portaria no 221, de 17 de abril de 2008. Publica a lista brasileira de internações por condições sensíveis à atenção primária. Diário Oficial da União, Brasília, p. 70, 18 abr. 2008. Seção 1. Disponível em: <http://bvsms.saude.gov.br/bvs/saudelegis/sas/2008/prt0221_17_04_2008.html>.

. Ministério da Saúde. Portaria n 3.242, de 30 de dezembro de 2011. Dispõe sobre o fluxograma laboratorial da Sífilis e utilização de testes rápidos para triagem da sífilis em situações especiais e apresenta outras recomendações. Diário Oficial da União, Brasília, 2011.

Protocolo Clinico e Diretrizes Terapêuticas para Atenção Integral às Pessoas com Infecções Sexualmente Transmissíveis. Brasília: Ministério da Saúde, 2015. 122p.

CAMPOS A.L.A.; ARAÚJO, M.A.L.; MELO, S.P.; ANDRADE, R.F.V.; GONÇALVES, M.L.C. Sífilis em parturientes: aspectos relacionados ao parceiro sexual. Revista Brasileira de Ginecologia e Obstetrícia, Fortaleza, v. 34, n. 9, p. 397-402, 2012. Disponível em: < http://www.scielo.br/pdf/rbgo/v34n9/a02v34n9.pdf>.

CARVALHO I. da S.; BRITO R. S. Sífilis congênita no Rio Grande do Norte: estudo descritivo do período 2007-2010. Epidemiologia e Serviços de Saúde, Brasília, v. 23, n. 1, p. 287-294, abr-jun, 2014. Disponível em: < http://www.scielo.br/pdf/ress/v23n2/1679-4974-ress-23-02-00287.pdf>. Doi: 10.5123/S1679-49742014000200010.

COSTA, C.C.; FREITAS, L.V.; SOUSA, D.M.N.; OLIVEIRA, L.L.; CHAGAS, A.C.M.A. et al. Sífilis congênita no Ceará: análise epidemiológica de uma década. Revista da Escola de Enfermagem da USP, São Paulo, v.47, n.1, p. 152-159, 2013. Disponível em: < http://www.scielo.br/pdf/reeusp/v47n1/a19v47n1>

CUNHA, A.R.C; HAMANN E.M. Sífilis em parturientes no Brasil: prevalência e fatores associados, 2010 a 2011. Revista Panamericana de Salud Pública, São Paulo, v. 38, n. 6, p. 479-486, 2015. Disponível em: < https://scielosp.org/pdf/rpsp/2015.v38n6/479-486/pt>.

DOMINGUES R.M.S.M.; LAURIA, L.M.; SARACENI, V.; LEAL, M.C. Manejo da sífilis na gestação: conhecimentos, práticas e atitudes dos profissionais pré-natalistas da 
rede SUS do município do Rio de Janeiro. Ciência e Saúde Coletiva, Rio de Janeiro, v. 18, n. 5, p. 1341-1351, 2013. Disponível em: < http://www.scielo.br/pdf/csc/v18n5/19.pdf>.

FIGUEIRÓ-FILHO, E.A.F.; FREIRE, S.S.A.; SOUZA, B.A.; AGUENA, G.S.; MAEDO, C.M. Sífilis e gestação: estudo comparativo de dois períodos (2006 e 2011) em população de puérperas. DST - Jornal Brasileiro de Doenças Sexualmente Transmissíveis, Mato Grosso, v. 24, n. 1, p. 32-37, 2012. Disponível em: < http://www.dst.uff.br/revista24-

12012/9.Sifilis\%20e\%20Gestacao\%20Estudo\%20Comparativo\%20de\%20Dois\%20P eriodos.pdf>. Doi: 10.5533/2177-8264-201224109.

GUANABARA M.A.O.; ARAÚJO, M.A.L; MATSUE, R.Y.; BARROS, V.L.; OLIVEIRA, F.A. Acesso de gestantes às tecnologias para prevenção e controle da sífilis congênita em Fortaleza-Ceará, Brasil. Revista de Salud Pública, v. 19, n. 1, '. 7378, 2017. Disponível em: < https://www.scielosp.org/article/rsap/2017.v19n1/7378/pt/>. Doi: 10.15446/rsap.v19n1.49295.

GUIMARÃES, T.A.; ALENCAR, L.C.R.; FONSECA, L.M.B.; GONÇALVES M.M.C.; SILVA, M.P. Sífilis em gestante e sífilis congênita no Maranhão. Arquivos de Ciências da Saúde, Maranhão, v. 25, n. 2, p. 24-30, 2018. Disponível em: < http://www.cienciasdasaude.famerp.br/index.php/racs/article/download/1023/759/>.

Doi:10.17696/2318-3691.25.2.2018.1023.

HOLANDA, M.T.C.G.; BARRETO, M.A.; MACHADO, K.M.M.; PEREIRA, R.C. Perfil epidemiológico da sífilis congênita no município de Natal, Rio Grande do Norte 2004 a 2007. Epidemiologia e Serviços de Saúde, Brasília, v.20, n.2, p. 203-212, 2011. Disponível em: < http://scielo.iec.gov.br/pdf/ess/v20n2/v20n2a09.pdf>. Doi: 10.5123/S1679-49742011000200009.

LAFETÁ K.R.G.; JÚNIOR, H.M.; SILVEIRA, M.F.; PARANAÍBA, L.M.R. Sífilis materna e congênita, subnotificação e difícil controle. Revista Brasileira de Epidemiologia, Minas Gerais, v. 19, n. 1, p. 63-74, 2016. Disponível em: < http://www.scielo.br/pdf/rbepid/v19n1/1980-5497-rbepid-19-01-00063.pdf.> Doi: 10.1590/1980-5497201600010006.

MAGALHÃES D.M.S.; KAWAGUCHI, I.A.L.; DIAS, A.; CALDERON, I.M.P. Sífilis materna e congênita: ainda um desafio. Caderno de Saúde Pública, Rio de Janeiro, v. 26, n. 6 , p. 1109-1120, 2013. Disponível em: < http://www.scielo.br/pdf/csp/v29n6/a08v29n6.pdf>.

MOREIRA, K.F.A.; OLIVEIRA, D.M.; ALENCAR, L.N.; CAVALCANTE, D.F.B.; PINHEIRO, A.S. et al. Perfil dos casos notificados de sífilis congênita. Cogitare Enfermagem, Rondônia, v. 22, n. 2, 2017. Disponível em: < https://revistas.ufpr.br/cogitare/article/download/48949/pdf>. Doi: 10.5380/ce.v22i1.48949.

PEREIRA, D.A.P.; MAIA, B.P.; SETO, I.I.C.; BICHARA, C..N.C. Infecção congênita em pacientes matriculados em programa de referência materno infantil. Revista 
Paraense de Medicina, v. 29, n. 1, 2015. Disponível em: < http://files.bvs.br/upload/S/0101-5907/2015/v29n1/a4655.pdf >.

ROSAS, D.S.A.; NOGUEIRA, M.C.M. Prevalência de sífilis congênita em Rio Branco - Acre nos anos de 2009 e 2013. 2017. 17p. Trabalho de Conclusão de Curso (Bacharelado em Enfermagem) - Centro Universitário UNINORTE, Rio Branco, 2017.

SÃO PAULO. Guia de bolso para o manejo da sífilis em gestantes e sífilis congênita. São Paulo: Secretaria de Estado de Saúde. 2016. 116p.

SIGNOR, M.; SPAGNOLO, L.M.L.; TOMBERG, J.O.; GOBATTO, M.; STOFEL, N.S. Distribuição espacial e caracterização de casos de sífilis congênita. Revista de enfermagem UFPE on line, Recife, v. 12, n. 2, p. 398-406, 2018. Disponível em: $<$ https://periodicos.ufpe.br/revistas/revistaenfermagem/article/download/230522/2784 4>. Doi: 10.5205/1981-8963-v12i2a230522p398-406-2018

SILVA, I.M.D.; LEAL, E.M.M.; PACHECO, H.F.; JÚNIOR, J.G.S.; SILVA, F.S. Perfil epidemiológico da sífilis congênita. Revista de enfermagem UFPE online, Recife, v. 13, n. 2, p. 604-623, 2019. Disponível em: < https://periodicos.ufpe.br/revistas/revistaenfermagem/article/view/236252/31536>.

Doi: 10.5205/1981-8963-v13i03a236252p604-613-2019.

SILVA, L.C.V.G.; TEODORO, C.J., SILVA, J.K.; SANTOS, D.A.S.; OLINDA, R.A. Perfil dos casos de Sífilis Congênita em um município do sul de Mato Grosso. Journal Health NPEPS. Mato Grosso, v. 2, n. 2, p. 380-390, 2017. Disponível em: < https://periodicos.unemat.br/index.php/jhnpeps/article/viewFile/2656/2148>.

SONDA, E.C.; RICHTER, F.F.; BOSCHETTI, G.; CASASOLA, M.P.; KRUMEL, C.F. et al. Sífilis Congênita: uma revisão da literatura. Revista de epidemiologia e controle de infecção, Rio Grande do Sul, v. 3, n. 1, p. 28-30, 2013. Disponível em: $<$ https://online.unisc.br/seer/index.php/epidemiologia/article/view/3022/2649 >.

SUTO C.S.S.; SILVA, DÉBORA, L.S.; ALMEIDA, E.S.; COSTA, L.E.L.; EVANGELISTA, T.J. Assistência pré-natal a gestante com diagnóstico de Sífilis. Revista de enfermagem e atenção à saúde, Bahia, v. 5, n. 2, p. 18-33, 2016. Disponível em:

http://seer.uftm.edu.br/revistaeletronica/index.php/enfer/article/download/1544/pdf>. 